\title{
Contribuição da FAPESP à Ciência e Tecnologia
}

\author{
ALBERTO CARVALHO DA SILVA
}

A

IDÉIA DE UMA FUNDAÇÃo de amparo à pesquisa surgiu em 1947 quando um grupo de homens de laboratório e de cátedra, liderado por dois engenheiros do IPT - Adriano Marchini e João Luiz Meiller - elaborou o documento Ciência e Pesquisa, no qual que se propunha incluir no texto da Constituição Estadual de 47, com base no artigo 174 da Constituição Federal de 1946 (1), um artigo estabelecendo que:

"É dever do Estado o amparo à Cultura e à Pesquisa Científica. Parágrafo primeiro - $\mathrm{O}$ amparo à pesquisa científica será exercido pelo Estado por intermédio de uma fundação, cujos moldes serão estabelecidos em estatutos aprovados pelo Governo. Parágrafo segundo Anualmente o Estado contribuirá para a fundação a que alude o parágrafo primeiro com quantia não inferior a $0,5 \%$ (meio por cento) do total de sua receita ordinária".

Após debates e contrapropostas, foi aprovado pela Assembléia Legislativa o Artigo 123 na Constituição de 1947, nos seguintes termos:

“Artigo 123-O amparo à pesquisa científica será propiciado pelo Estado por intermédio de uma fundação organizada nos moldes que forem estabelecidos por lei. Parágrafo único-Anualmente o Estado atribuirá a essa fundação, como renda especial de sua privativa administração, quantia não inferior a meio por cento do total de sua receita ordinária”.

\section{Instalação da Fundação}

Nesse mesmo ano foram elaborados os primeiros projetos de lei para a nova fundação (2), inspirados nos Fundos Universitários de Pesquisa (3). Por sugestão de Marchini e Meiller, o reitor da USP (4) nomeou uma comissão para elaborar um anteprojeto que foi aprovado pelo Conselho Universitário e encaminhado ao governador (5), mas sem resultado. Com a criação da SBPC, reforçou-se a campanha das lideranças científicas para que a fundação fosse instalada, inclusive com apresentação de novo anteprojeto. A criação do CNPq e da CAPES pelo governo federal, em 1951, trouxe novo estímulo à campanha em São Paulo e em 1954, já ao fim de seu mandato, o governador (6) submeteu ao Conselho Universitário uma nova versão, mas também sem resultado.

O governador seguinte (7) teve desentendimentos com a USP e não demonstrou 
interesse pela fundação apesar dos esforços continuados da SBPC e da Associação de Auxiliares de Ensino da Universidade de São Paulo (8). Finalmente, ao assumir o governo do estado em 1959, Carvalho Pinto, acolhendo as manifestações da SBPC, da associação dos docentes e de outras entidades, nomeou uma comissão para elaborar um anteprojeto de lei regulamentando a fundação (9). Neste trabalho foi fundamental a contribuição de um dos membros da comissão, Paulo Vanzolini, por sua experiência científica, por seu conhecimento sobre as formas de apoio à pesquisa nas fundações Ford, Guggenheim e Rockefeller e no Instituto Max Plank e pela análise do desempenho do CNPq, já então com oito anos de atividade (10).

Em setembro de 1959 Carvalho Pinto submeteu ao Conselho Universitário o anteprojeto, que foi aprovado com alguns acréscimos e, em 18 de outubro de 1969, a Assembléia Legislativa aprovou a Lei 5.918 instituindo a Fundação de Amparo à Pesquisa de São Paulo (FAPESP).

A instalação ficou a cargo do reitor da USP, Antonio Barros de Ulhoa Cintra, nomeado presidente do Conselho Superior (11). Depois de 18 meses, que foram necessários para compor esse Conselho, escolher e nomear os três diretores do Conselho Técnico Administrativo (12), montar a infra-estrutura de administração e elaborar e aprovar os estatutos, a Fundação iniciou as suas atividades em 1 de junho de 1962. Instalada a Fundação, Carvalho Pinto determinou a transferência de recursos extras equivalentes a US\$ 5.7 milhões, correspondentes à dotação que lhe caberia no período 1956-1960, iniciando assim a formação de seu patrimônio em obediência ao artigo $3^{\circ}$ da Lei 5.918 (13).

Coube ao seu primeiro diretor científico, Warwick Kerr, organizar a Diretoria Científica, escolher o corpo de assessores e estabelecer os procedimentos de análise e decisão sobre as propostas recebidas, adotando o sistema de pareceres sigilosos por pesquisadores com experiência na área correspondente ao pedido (peer review) (14).

\section{Instrumentos de apoio à pesquisa e para a formação de pesquisadores}

As ações da FAPESP foram, desde o início, divididas em bolsas e auxílios propostos diretamente pelos interessados e seguindo normas que até hoje são mantidas: as bolsas são concedidas em função de projetos de pesquisa avaliados quanto ao seu mérito científico e ao curriculum do orientador; os auxílios são destinados a financiar projetos de pesquisa, convites para especialistas nacionais e estrangeiros e, ainda, para organização e realização de reuniões científicas e publicação de trabalhos.

Sem prejuízo dessas normas, a Fundação vem procurando promover a cooperação entre diversos pesquisadores, identificar e estimular a pesquisa em áreas do conhecimento e temas que, por seu interesse científico ou social, mereçam prioridade. Dentro dessa estratégia enquadram-se as iniciativas, os projetos especiais e os projetos temáticos de equipe. 
Mais recentemente, tendo em conta a necessidade de se estimular o desenvolvimento tecnológico e a inovação na empresa, a Fundação lançou, em 19941995, o Programa de Apoio à Capacitação Tecnológica das Universidades, Instituições de Pesquisa e Desenvolvimento de Empresas: Projeto de Inovação Tecnológica em Parceria. Também, considerando que as universidades e institutos estão atualmente encontrando dificuldade em renovar e manter instalações e serviços em condições adequadas para as suas atividades de pesquisa, a Fundação aprovou, em 1995, em caráter de emergência, o Programa de Apoio à Recuperação e Modernização de Infra-Estrutura de Pesquisa do Sistema Estadual de Ciência e Tecnologia.

\section{Programas de bolsas e auxilios individuais}

Desde a sua instalação até 31 de dezembro de 1995, a Fundação recebeu 59.960 pedidos de bolsas e 51.600 pedidos de auxílios, aprovando, respectivamente, 40.825 e 32.071, conforme demostrado no Quadro 1 (15).

Quadro 1

Número de bolsas e auxílios aprovados até 31 de dezembro de 1995

\begin{tabular}{|c|c|c|c|}
\hline Bolsas & & Auxílios & \\
\hline Total aprovado & 40.825 & Total aprovado & 32.071 \\
\hline Inic. científica & $31.7 \%$ & Projetos de pesquisa & $53.0 \%$ \\
\hline Aperfeiçoamento ${ }^{(a)}$ & $10.6 \%$ & Prof. Visitante do Brasil & $11 \quad 0.9 \%$ \\
\hline Mestrado $^{(b)(d)}$ & $26.5 \%$ & Prof. Visitante do Ext. & $10.2 \%$ \\
\hline Doutorado I e II ${ }^{(\mathrm{d})}$ & $13.8 \%$ & Par. Reunião Cient. Br. & $7.4 \%$ \\
\hline Pós-doutorado & $2.8 \%$ & Par. Reunião Cient. Ex. & $15.3 \%$ \\
\hline \multirow[t]{2}{*}{ Exterior $(\mathrm{PG} \text { e } \mathrm{PD})^{(\mathrm{c})}$} & $14.5 \%$ & Org. Reun.Cient. & $8.5 \%$ \\
\hline & & Publicações & $4.7 \%$ \\
\hline
\end{tabular}

(a) Até 1977 as bolsas de mestrado eram classificadas como aperfeiçoamento; ${ }^{\text {(b) }}$ Até 1980 auxílios para convite a professores visitantes de outras regiões do Brasil eram classificados como auxílios à pesquisa; (c) A partir de 1980, quando as bolsas no exterior foram divididas em doutorado e pós-doutorado, foram aprovadas 859 de doutorado e 3.249 de pósdoutorado; (d) A maior parte das bolsas de mestrado e doutorado no estado de São Paulo são concedidas por CNPq e CAPES.

Merece destaque a ênfase dada pela Fundação à iniciação científica, que dá conta de quase um terço de todas as bolsas aprovadas, e cuja contribuição para a pósgraduação e a formação de pesquisadores foi comprovada em alguns estudos (16).

Outros estudos confirmam que os instrumentos utilizados pela FAPESP estão alcançando os resultados desejados. Um levantamento da produção científica de 2.190 pesquisadores e bolsistas que, entre 1980 e 1985 receberam auxílios da Fundação, mostrou um total de 3.404 trabalhos em revistas científicas, 7.005 apre- 
sentações em reuniões científicas e congressos, 154 livros, 306 capítulos em livros e 967 dissertações e teses, relacionados com os auxílios e bolsas aprovados.

A evolução do número de bolsas e auxílios solicitados e aprovados entre 1962 e 1995 é apresentada em triênios na figura 1. Em média, para todo o período, foram aprovados $62,1 \%$ dos pedidos de bolsas e $68 \%$ dos pedidos de auxílios, mas com apreciáveis variações por triênio. Os mínimos foram 53,4\% para auxílios em 1993-1995 e 61,5\% para bolsas, em 1990-1992; os máximos, 81,7\% para auxílios em 1966-1968 e 76,4\% para bolsas em 1975-1977 (17).

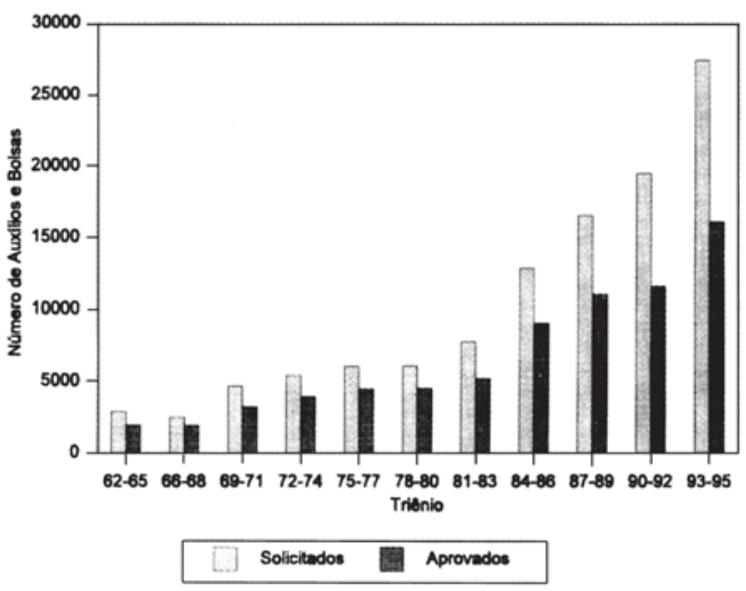

Figura 1

Número de auxílios e bolsas solicitadas e aprovadas, por triênio, entre 1962 e 1995. O primeiro triênio inclui o segundo semestre de 1962.

\section{Iniciativas}

Desde o início, a Fundação vem mantendo, sob o título de Iniciativas, auxílios em que, identificando lacunas no conhecimento, procura assegurar meios para o seu preenchimento.

Em um conjunto de 24 iniciativas a partir de 1962, algumas destacam-se por seu interesse científico, social ou econômico (18):

- Estudos sobre cancro cítrico 1963 - Sob a direção de Victoria Rossetti, o Instituto Biológico, que contribuiu para erradicação dessa doença dos laranjais.

- Laboratório de Biotecnologia Industrial 1968 - Escola Politécnica da USP, com participação da Faculdade de Ciências Farmacêuticas, Instituto de Pesquisas Tecnológicas, Escola de Engenharia Mauá e Instituto de Pesquisas Energéticas e Nucleares.

- Levantamentos biológicos em base ecológica do litoral e da área costeira 1966 - Desenvolvido na baía da Ilha Grande pelo Instituto Oceanográfico 
da USP, em colaboração com Instituto de Botânica e Departamentos de Geologia e Geografia e com a participação da Secretaria da Agricultura.

- Levantamento faunístico, ecológico e econômico dos recursos pesqueiros do Amazonas 1966 - Departamento de Zoologia da Secretaria de Agricultura de São Paulo, Instituto Nacional de Pesquisa da Amazônia, Museu Goeldi e Universidade de Harvard.

- Interpretação científica da paisagem 1966- Fotografias aéreas e trabalho de campo abrangendo área ao longo da rodovia Rio-Bahia, Raso de Santa Catarina e Paulo Afonso, em uma extensão de 5 mil km, com cerca de 600 fotos como documentação para seminário sobre o tema.

- Estudos sobre materiais pré-fabricados para construções escolares de baixo custo 1966 - Escola Politécnica da USP.

- Laboratório de Micro-eletrônica 1968-Departamento de Engenharia de Eletricidade e Laboratório de Semicondutores da Escola Politécnica da USP.

- Programa multidisciplinar de nutrição 1974-Em colaboração com CNPq, CAPES e Fundação Ford, para pesquisa e treinamento em nutrição humana, em apoio ao Programa Nacional de Nutrição (PRONAN), implantado em 1973 pelo governo federal em cooperação com o Banco Mundial.

Embora não planejado como iniciativa, também merece destaque o apoio dado ao Instituto de Pesquisas Tecnológicas (IPT), desde 1962, com a aprovação, até 1995, de 120 auxílios, 42 bolsas no país e 33 no exterior, abrangendo trabalho sobre madeiras comerciais, celulose e papel, fundição de precisão, materiais cerâmicos, biotecnologia, dinâmica de flutuantes e construções oceânicas.

\section{Projetos Especiais}

Entre 1970 e 1988, a Fundação lançou sete projetos especiais atendendo a propostas de grupos de pesquisadores, na maioria dos casos filiados a diferentes instituições. Para a análise de várias propostas a FAPESP recorreu a consultoria de especialistas do exterior.

- Projeto Bioq. FAPESP 1970 - Em apoio do desenvolvimento da bioquímica. Apresentado pelo Instituto de Química da USP e pelo Departamento de Bioquímica da Escola Paulista de Medicina (19). Contribuiu para o desenvolvimento de laboratórios de sintese de peptídeos, espectropolarimetria e instrumentação eletrônica; até 1985, foram publicados 394 trabalhos pelos grupos participantes.

- Meteorologia com radar (RADASP I e II) 1974 - O projeto partiu de um parecer do professor James A. Weiman, da Universidade de Wisconsin, convidado pela FAPESP para avaliar as oportunidades de apoio a essa linha de pesquisa. O RADASP contribuiu para a instalação de radar no Instituto de Pesquisas Meteorológicas, Fundação Educacional de Bauru, posteriormente incorporado à UNESP. Face às aplicações na agricultura e 
outros setores da economia, com a colaboração de INPE, DAEE, Escola de Engenharia de Ilha Solteira (UNESP) e Escola Politécnica da USP, o projeto expandiu-se, procedendo-se a estudos sobre chuvas de verão e camadas da atmosfera, com o uso de radio sondas, balões cativos, planadores e aviões, estabelecendo-se ampla colaboração científica com Canadá, França, Estados Unidos, Portugal e Reino Unido. Em 1986 foi instalado um segundo radar na barragem do DAEE em Ponte Nova.

- Tipologia das represas do estado de São Paulo 1978 - Laboratório de Limnologia/UFSCar, Departamento de Ecologia/Instituto de Biociências da USP e Instituto de Pesca da Secretaria de Agricultura do Estado de São Paulo. Resultou numa produção científica de 150 trabalhos publicados no Brasil e no exterior, quatro livros, sendo três no exterior, dez teses de doutorado e 15 dissertações de mestrado. Deu origem a iniciativas semelhantes em Minas Gerais e Paraná, contribuindo para a formação de conhecimentos sobre mecanismos de funcionamento de represas.

- Ação Programada em águas subterrâneas 1982 - Em colaboração com o Departamento de Águas e Energia Elétrica (DAEE), Departamento de Hidráulica da Escola de Engenharia de São Carlos da USP, Instituto de Geociências de Rio Claro-UNESP, Departamento de Geologia, Física e Matemática em Ribeirão Preto da USP, Instituto de Geociências da USP, teve considerável produção científica.

- Centro de bioterismo 1984-Resultado de uma proposta de pesquisadores da USP, UNICAMP e Escola Paulista de Medicina, pleiteando o apoio da FAPESP para a produção e manutenção de animais usados em experimentação e controle, dentro de condições sanitárias genéticas e ambientais padronizadas. Com base no parecer de especialistas nacionais e do exterior convidados pela Fundação, foi aprovado o apoio a três entidades, uma das quais a UNICAMP, atualmente, constitui-se centro de referência para treinamento, transferência e desenvolvimento de tecnologias e produção, manutenção e fornecimento de várias linhagens de animais axênicos, gnotobióticos e livres de agentes patogênicos (SPF).

- Laboratório de tratamento de imagens 1986 - Instituto Astronômico e Geofísico da USP. Numerosos projetos científicos estão se beneficiando do laboratório, que tem sido usado no tratamento de imagens de diversas procedências.

- Rede ANSP 1988 - A rede ANSP (Academic Network at São Paulo), resultou de uma proposta de pesquisadores das três universidades mantidas pelo estado (USP, UNICAMP, UNESP). Em 1988 ligou-se à Bitnet e Hepnet através de uma linha entre FAPESP e Fermilab. A partir de 1992, ligou-se à Internet. Em nível nacional passou a interligar-se com as demais regiões do país através da RNP (Rede Nacional de Pesquisa-CNPq), com linhas diretas de 64 kbps para Brasília, Rio de Janeiro e Rio Grande do Sul e a 9.6 kbps para Minas Gerais, Pernambuco e Paraná. Em 1994 o canal internacional foi expandido para $128 \mathrm{kbps}$ dobrando novamente em 1995. Até o fim de 1996 estão previstos cerca de 16.500 pontos nas três 
universidades do estado, sendo cerca de 8 mil na USP, 3.500 na UNICAMP e 5 mil na UNESP. Atualmente estão implantadas ligações com 17 cidades do estado, prevendo-se mais 14 em futuro próximo.

Tendo em vista os resultados anteriores, em 1988 foi anunciado um amplo programa de Projetos Especiais visando a estimular estudos de maior interesse científico e social por pesquisadores ou grupos de pesquisadores de um ou mais centros de pesquisa. Foram recebidas 164 propostas e aprovadas 72 , a um custo total de US\$ 14,3 milhões, metade delas nas áreas de Física e Ciências da Saúde, conforme quadro 2.

Quadro 2

Projetos especiais aprovados em 1989

Distribuição por área de conhecimento e valor em dólares

\begin{tabular}{lrr}
\hline Área de conhecimento & Número & $\begin{array}{c}\text { Projetos aprovados } \\
\text { custo em dólares }\end{array}$ \\
\hline Agrária & 2 & 271.991 \\
Biológicas & 7 & 1.525 .114 \\
Ecologia & 1 & 1.224 .541 \\
Economia-administração & 1 & 58.667 \\
Engenharia & 9 & 1.042 .401 \\
Física & 18 & 4.696 .290 \\
Geociências & 3 & 338.724 \\
Humanas e Sociais & 3 & 412.595 \\
Interdisciplinar & 1 & 42.873 \\
Matemática-estatística & 2 & 60.284 \\
Química & 9 & 2.655 .248 \\
Saúde & 16 & 1.974 .483 \\
Totais & 72 & 14.303 .211 \\
\hline
\end{tabular}

Oitenta e cinco por cento dos projetos desenvolveram-se na USP (57.4\%), UNICAMP (15.8\%) e institutos de pesquisa do estado (11.8\%). UNESP e USFCar somaram 11.2\%, seguidos da Escola Paulista de Medicina com 3.5\%. O programa estendeu-se até 1992, quando foram encerrados os últimos projetos. Entre os resultados obtidos destacam-se: consolidação do grupo de geoquímica na USP; aumento da capacidade de síntese de peptídeos de interesse em biotecnologia na Escola Paulista de Medicina; ampliação, reorganização e renovação de equipamentos das casas de vegetação nos Institutos de Botânica, Agronômico e Florestal e nas três universidades do estado; desenvolvimento das etapas preparatórias para o uso de cultura de tecidos na industrialização da vacina anti-rábica no Instituto Butantan; instalação de um laboratório de econometria na USP; maiores recursos para análise estatística em agropecuária no Instituto Agronômico; montagem de laboratório de ótica para estudo de semicondutores no Centro de Ciências Tecnológicas-UfSCar; estudos sobre solos no ITA; Centro de 
Espectroscopia a Laser na USP-São Carlos; novas tecnologias em laser de $\mathrm{Co}_{2}$ na UNICAMP.

\section{Projetos temáticos de equipe}

O programa foi lançado em 1990, com o objetivo de intensificar a cooperação entre pesquisadores de diferentes grupos, departamentos, unidades ou instituições, encorajando-se a participação de várias áreas disciplinares sob coordenação de um pesquisador responsável pelo projeto. Desde 1990 até setembro de 1996 foram aprovados 240 projetos, a um custo de US\$ 57 milhões, além de suplementações no valor de US\$ 73 milhões.

O quadro 3 apresenta os investimentos no período 1990-1995. Os projetos são distribuídos por área de conhecimento, mas deve ser levado em conta que esta corresponde à área do coordenador do projeto, podendo os demais serem de outras.

Quatro áreas - engenharia, física, química e saúde - dão conta de $68 \%$ do total investido, contribuindo com $55 \%$ dos projetos. As três universidades estaduais dão conta de $76 \%$ dos projetos; $9 \%$ cabem aos institutos de pesquisa do estado; $12 \%$ ao INPE e às duas universidades federais do estado, UFSCar e UFSP; $\mathrm{e}$ $3 \%$ a entidades particulares de ensino e pesquisa.

\section{Quadro 3}

Projetos temáticos de equipe até setembro de 1996.

Número de projetos e orçamento aprovados por área do conhecimento.

\begin{tabular}{lcc}
\hline Área de conhecimento $^{(\mathrm{a})}$ & Projetos aprovados & Recursos $^{(\mathrm{b})}$ US\$ x 1.000 \\
\hline Agronomia e Veterinária & 14 & 1.131 .9 \\
Arquitetura e Urbanismo & 3 & 584.5 \\
Astronomia-Ciên. do Espaço & 3 & 819.5 \\
Engenharias & 34 & 7.863 .6 \\
Física & 27 & $6.872,6$ \\
Geociências & 12 & $3.464,4$ \\
Ciências humanas e sociais & 19 & $1.569,7$ \\
Ciências biológicas & 29 & $10.022,6$ \\
Matemática & 6 & 641,5 \\
Química & 26 & 8.926 .3 \\
Ciências da saúde & 43 & $10.807,0$ \\
Temáticos & $(\mathrm{c})$ & 4.437 .2 \\
Totais & 24 & $57.140,8$ \\
\hline
\end{tabular}

(a) Área do coordenador do projeto; (b) Não incluem suplementações que somam US\$ 73 milhões; ${ }^{(c)}$ Projetos designados como temáticos independentemente da área do coordenador. 


\section{Programa de Apoio à Capacitação Tecnológica das Universidades, Institutos de Pesquisa e Desenvolvimento de Empresas: Projeto de Inovação Tecnológica em Parceria.}

A FAPESP lançou, a partir de 1995, um programa de apoio a projetos desenvolvidos em parceria universidade ou institutos de pesquisa com empresas. Os recursos concedidos pela FAPESP são destinados aos centros de pesquisa, cabendo às empresas contribuir com os recursos próprios adicionais que podem ser obtidos junto a outras agências de fomento ou sob a forma de incentivos fiscais. A proposta deve ser apresentada em conjunto pelas entidades participantes. Os resultados do projeto pertencem às entidades proponentes, acertados entre elas, com o aval da FAPESP. Em 1995, foram aprovadas nove propostas e outras seis estão em análise. Em 1994 foram alocados ao programa R 5 milhões e, em 1995, R\$ 10 milhões (20).

\section{Programa de Apoio à Recuperação e Modernização da Infra-Estrutura de Pesquisa do Setor Estadual de Ciência e Tecnologia do Estado de São Paulo}

O programa, introduzido no segundo semestre de 1994, tem por objetivo conceder auxílios a grupos de pesquisa para financiamento de itens de infra-estrutura não incluídos nos auxílios da Fundação em sua forma tradicional. Até dezembro de 1995 foram aprovados 847 pedidos, com um investimento deR 66 milhões, abrangendo todas as áreas de conhecimento (quadro 4).

Quadro 4

Programa Emergencial de Apoio à Recuperação da Infra-Estrutura de Pesquisa no Sistema Estadual de Ciência e Tecnologia. Distribuição por área de conhecimento.

\begin{tabular}{lcrrr}
\hline Área do conhecimento & $\begin{array}{l}\text { Investimentos } \\
\text { em reaisdo }\end{array}$ & $\begin{array}{l}\text { em \% } \\
\text { total }\end{array}$ & $\begin{array}{l}\text { Número } \\
\text { de projetos }\end{array}$ & $\begin{array}{c}\% \text { do } \\
\text { total }\end{array}$ \\
\hline Arquitetura e urbanismo & 219.5 & 0.33 & 3 & 0.35 \\
Astronomia e ciências espaciais & 185.6 & 0.28 & 3 & 0.35 \\
Ciências agrárias & 9.437 .4 & 14.28 & 117 & 13.81 \\
Ciêcias biológicas & 5.498 .7 & 8.32 & 74 & 8.74 \\
Ciências da saúde & 12.527 .5 & 18.96 & 189 & 22.31 \\
Econômicas e administrativas & 233.8 & 0.35 & 7 & 0.83 \\
Ciências humanas e sociais & 8.311 .7 & 12.58 & 81 & 9.56 \\
Engenharia & 9.587 .9 & 14.51 & 171 & 20.19 \\
Física & 6.646 .7 & 10.06 & 77 & 9.10 \\
Geociências & 2.146 .5 & 3.25 & 39 & 4.61 \\
Matemática & 3.591 .1 & 5.44 & 25 & 2.95 \\
Química & 7.691 .7 & 11.64 & 61 & 7.20 \\
Total & 66.078 .1 & 100.00 & 847 & 100.00 \\
\hline
\end{tabular}


O quadro 5 mostra distribuição por instituição. O programa foi aprovado em caráter de emergência, tendo em vista a necessidade de manter em boas condições as instalações básicas dos laboratórios, atualizar e conservar equipamentos de uso geral, manter em condições apropriadas biotérios e outros serviços de apoio à experimentação e controle; atualizar bibliotecas e corrigir falhas nas coleções. Das 12 áreas de conhecimento atendidas pelo Programa, seis receberam, cada uma, entre 10\% e 19\% dos recursos, totalizando $82 \%$.

Quadro 5

Programa Emergencial de Apoio à Recuperação e Modernização da Infra-Estrutura de Pesquisa do Sistema Estadual de Ciência e Tecnologia. Distribuição por instituição

\begin{tabular}{lcc}
\hline $\begin{array}{l}\text { Vínculo institucional } \\
\text { do pesquisador }\end{array}$ & $\begin{array}{l}\text { Número } \\
\text { de projetos }\end{array}$ & $\begin{array}{l}\text { Projetos aprovados } \\
\text { (US\$ x 1.000) }\end{array}$ \\
\hline USP & 240 & 31.046 .1 \\
Unesp & 231 & 11.451 .5 \\
Unicamp & 165 & 10.874 .5 \\
Inst. das secretarias de estado & 103 & 6.085 .6 \\
Entidades federais & 90 & 5.840 .8 \\
Ent. partic. de ensino e pesquisa & 17 & 683.9 \\
Ent. partic. de pesquisa & 1 & 95.8 \\
Totais & 847 & 66.078 .2 \\
\hline
\end{tabular}

\section{Convênios com entidades de outros países}

A FAPESP mantém acordos de intercâmbio com Fundação Fullbright, Conselho Britânico, Fundação Von Humboldt, Serviço Alemão de Intercâmbio Acadêmico (DAAD), Junta Nacional de Investigação Científica e Tecnológica de Portugal (JNICT) e Comitê Estatal de Colaboração Econômica de Cuba. De 1992 a 1995 esse intercâmbio propiciou a visita de 76 pesquisadores de centros do exterior a São Paulo e de 66 pesquisadores de São Paulo a centros do exterio. (quadro 6).

Quadro 6

Convênios da FAPESP com entidades de outros países Período 1991-1995

\begin{tabular}{lcccc}
\hline \multicolumn{5}{c}{ Intercâmbios } \\
Entidade & de São Paulo para Exterior & do Exterior para São Paulo & Total \\
\hline British Council & 11 & 24 & 35 \\
Daad & 41 & 38 & 79 \\
Fund. Fullbright & 2 & 1 & 3 \\
Von Humboldt & 6 & 1 & 7 \\
JNCIT & 6 & 12 & 18 \\
Total & 66 & 76 & 142 \\
\hline
\end{tabular}


Por meio da participações em reuniões científicas, professores visitantes e bolsistas de doutorado e pós-doutorado, a FAPESP propiciou, entre 1991 e 1995, intercâmbio com cerca de 60 países, com um total de 4.887 açãoes aprovadas (quadro 7).

Quadro 7

Intercâmbio com outros países - 1991 a 1995

\begin{tabular}{|c|c|c|c|c|c|}
\hline & & & Modal & idade & \\
\hline Reuniõ & óes científicas & Visitantes & Doutorados & Pós-Doutorados & Total \\
\hline Países & & & & & \\
\hline EUA & 610 & 291 & 103 & 628 & 1.632 \\
\hline França & 132 & 125 & 14 & 194 & 465 \\
\hline Reino Unido & 108 & 97 & 12 & 127 & 344 \\
\hline Alemanha & 89 & 65 & 4 & 77 & 235 \\
\hline Itália & 128 & 72 & 2 & 89 & 291 \\
\hline Canadá & 119 & 52 & 3 & 61 & 235 \\
\hline Japão & 87 & 39 & 4 & 22 & 152 \\
\hline Portugal $^{(1)}$ & 96 & 28 & 1 & 26 & 151 \\
\hline Argentina & 76 & 37 & 4 & - & 117 \\
\hline Espanha & 116 & 32 & 5 & 33 & 186 \\
\hline México ${ }^{(2)}$ & 62 & 15 & 2 & 79 & 158 \\
\hline Austrália ${ }^{(2)}$ & 44 & 8 & 1 & 5 & 58 \\
\hline Chile $^{(3)}$ & 34 & 9 & - & 3 & 46 \\
\hline Outros $^{(4)}$ & 491 & 197 & 13 & 116 & 817 \\
\hline Total & 2.192 & 1.067 & 168 & 1.460 & 4.887 \\
\hline
\end{tabular}

(1) Não inclui 1991; (2) Não inclui 1991 e 1992; (3) Não inclui 1991, 1992, 1993; ${ }^{(4)} 24$ países em 1991; 43, em 1992; 39, em 1993; 38, em 1994; 46, em 1995.

\section{Dotação financeira}

As transferências de recursos pelo estado à FAPESP, entre 1962 e 1965, somam o equivalente a cerca de US\$ 750 milhões e podem ser divididas em três fases $(21)$ :

- Desde a instalação até 1984 , quando os recursos eram transferidos uma ou duas vezes por ano. Deduzidas as transferências aos municípios e os efeitos da inflação, os recursos do estado corresponderam, em média, a $0,217 \%$ da receita ordinária e não a $0,5 \%$ como estabeleceu a Constituição de 1947. O total transferido durante esse período, excluídos os US\$ 5.7 milhões correspondentes aos exercícios de 1956 a 1960, foi da ordem de US\$ 150 milhões.

- De 1985 até 1989, de acordo com a emenda 39 de autoria do deputado 
Fernando Leça, as transferências passaram a ser mensais, calculadas em duodécimos da arrecadação prevista. O total transferido no período foi de ordem de US\$ 120 milhões.

- De 1990 em diante, quando, pela Constituição Estadual de 1989, a dotação passou a ser transferida mensalmente, calculada na base de $1 \%$ da renda tributária do mês anterior e deduzidas as transferências aos municípios. Para o período de 1990-1995, a dotação somou a cerca de US\$ 480 milhões.

No total, as médias anuais das transferências foram da ordem de US\$ 7 milhões até 1984; US\$ 24 milhões entre 1985 e 1989; e US\$ 80 milhões entre 1990 e 1995, mas com acentuado crescimento anual entre 1991 US\$ 51 milhões e 1995 US\$ 151 milhões.

Em média, e com pequenas variações entre triênios, a partir de 1978, 82\% dos recursos investidos em auxílios e bolsas concentram-se em sete áreas de conhecimento (22) entre as 13 áreas (23) adotados pela FAPESP (quadro 8).

Quadro 8

Distribuição do investimento em auxílios e bolsas nas sete áreas que absorvem $5 \%$ ou mais recursos Média anual em percentual do total, a partir de 1978

\begin{tabular}{lrrrrrrr}
\hline & \multicolumn{7}{c}{ Triênios } \\
Área & $78 / 80$ & $81 / 83$ & $84 / 86$ & $87 / 89$ & $90 / 92$ & $93 / 95$ & Médias \\
\hline Agronomia & 2.50 & 3.79 & 4.89 & 7.06 & 7.33 & 8.55 & 5.69 \\
Biologia & 21.90 & 18.39 & 12.93 & 9.44 & 16.29 & 11.39 & 15.06 \\
Física & 14.19 & 12.48 & 13.91 & 16.94 & 14.71 & 11.94 & 14.03 \\
H. Sociais & 10.03 & 11.85 & 12.75 & 9.25 & 5.87 & 7.35 & 9.52 \\
Saúde & 11.78 & 14.85 & 7.29 & 17.92 & 18.85 & 21.47 & 17.03 \\
Química & 11.25 & 9.73 & 10.26 & 11.40 & 8.43 & 10.70 & 10.29 \\
Engenharia & 10.10 & 11.12 & 8.52 & 7.28 & 12.19 & 13.74 & 10.49 \\
Soma & 81.75 & 82.21 & 80.55 & 79.29 & 83.67 & 85.19 & 82.11 \\
\hline
\end{tabular}

Entre 1978 e 1995, as três universidades estaduais absorveram 80.4\% dos recursos, cabendo 55.6\% à USP, porém, com nítida tendência a diminuir. A UNICAMP, com $16.5 \%$ apresentou um pequeno aumento, enquanto a UNESP teve, em média, 8.3\%, e mais do que dobrou a sua participação entre 1978 e 1995 (quadro 9).

\section{Comentários e conclusões}

Graças à continuidade com que a FAPESP vem atuando e à regularidade com que o governo vem transferindo recursos, de acordo com o estabelecido nas Constituições Estaduais de 1947 e 1989, a atividade científica de São Paulo, 
Quadro 9

Distribuição do investimento em auxílios e bolsas por instituição

Média anual por triênio em percentual do total, a partir de 1978

\begin{tabular}{|c|c|c|c|c|c|c|c|}
\hline \multirow[b]{2}{*}{ Instituição } & \multicolumn{7}{|c|}{ Triênios } \\
\hline & $78 / 80$ & $81 / 83$ & $84 / 86$ & $87 / 89$ & $90 / 92$ & 93/95 & Médias \\
\hline USP & 60.06 & 58.36 & 57.12 & 55.44 & 52.41 & 49.24 & 55.44 \\
\hline Unicamp & 15.63 & 14.62 & 17.52 & 17.25 & 15.90 & 18.12 & 16.52 \\
\hline Unesp & 5.88 & 5.67 & 6.41 & 8.25 & 8.25 & 14.77 & 8.20 \\
\hline Entid. Fed. ${ }^{(a)}$ & 6.57 & 9.02 & 9.41 & 10.03 & 11.71 & 9.24 & 9.33 \\
\hline Ins.Pesq.Est. ${ }^{(b)}$ & 3.72 & 3.49 & 3.82 & 4.59 & 8.49 & 5.13 & 4.87 \\
\hline En.Par.Ens.Pes & s. 3.47 & 4.73 & 4.25 & 3.07 & 3.25 & 2.35 & 3.52 \\
\hline Ent. Munic. & 0.14 & 1.91 & 0.10 & 0.29 & 0.06 & 0.07 & 0.41 \\
\hline Fimas Part. & 0.44 & 0.10 & 0.09 & 0.06 & 0.06 & 0.81 & 0.26 \\
\hline Pessoa Física & 1.26 & 2.01 & 1.24 & 0.57 & 0.43 & 0.21 & 0.88 \\
\hline Outros $^{(c)}$ & 2.83 & 0.09 & 0.04 & 0.45 & - & 0.06 & 0.58 \\
\hline Total & 100.00 & 100.00 & 100.00 & 100.00 & 100.00 & 100.00 & 100.00 \\
\hline
\end{tabular}

(a) Universidade Federal de São Carlos, Universidade Federal Paulista (Escola Paulista de Medicina), Instituto Nacional de Pesquisas Espaciais, Instituto de Tecnologia da Aeronáutica, Instituo de Pesquisas Energéticas e Nucleares; ${ }^{(b)}$ No total, 17; ${ }^{(c)}$ Itens abrangendo mais de uma instituição, como projetos interdisciplinares, iniciativas, simpósios etc.

antes concentrada na capital, vem se desenvolvendo satisfatoriamente e expandindo-se para outros centros, principalmente Campinas, São Carlos, Ribeirão Preto, Piracicaba e São José dos Campos, Botucatu. Entre outros indicadores, comprovam este desenvolvimento a grande concentração no estado de programas de pós-graduação em nível A e B, de pesquisadores em nível de doutorado e de publicações científicas em revistas com corpo editorial seletivo.

O maior desenvolvimento de São Paulo em P\&D lhe dá considerável vantagem na captação dos recursos federais. São Paulo absorve um terço dos recursos federais para pós-graduação no país e no exterior; $35 \%$ dos recursos do PADCT e FINEP. Embora a descentralização da capacidade em P\&D seja crucial para o equilíbrio no desenvolvimento econômico e social do país, continua prevalecendo um círculo vicioso em que os centros mais ricos disputam com vantagem cada vez maior os recursos escassos, agravando-se a desigualdade em vez de diminuir.

A política para se conseguir a descentralização reside no fortalecimento da capacidade regional de pesquisa e incorporação de novos pesquisadores. Nesse sentido, a implantação da FAPESP em 1960 encorajou iniciativas semelhantes no Rio Grande do Sul (FAPERGS, 1964), Rio de Janeiro (FAPERJ, 1980), Minas Gerais (FAPEMIG, 1985). Mas, na falta de dispositivo constitucional, nenhuma das três fundações obteve o sucesso da FAPESP. 
Com base do parágrafo $5^{\circ}$ no Artigo 128 da Constituição Federal de 1988 (24), 22 estados e o Distrito Federal incluíram em suas Constituições a criação de fundações ou fundos, com vinculações entre $0.3 \%$ e $3 \%$ com diversas bases de cálculo (25).

Calculou-se, em 1990, que se todos os estados e DF implantassem as suas fundações ou fundos e transferissem os recursos previstos, o total seria de US\$ 340 milhões, dos quais US\$2 20 milhões caberiam a FAPERGS, FAPEMIG, FAPESP e FAPERJ. Todavia, até 1995, apenas 14 estados e o DF haviam cumprido com suas Constituições e, apesar da previsão ser de cerca de US\$ 250 milhões excluída a FAPESP, apenas cerca de US\$ 40 milhões, aproximadamente $16 \%$ do devido, foram transferidos nesse ano (26).

Como indica o exemplo da FAPESP, a obediência dos estados às suas Constituições favoreceria o seu desenvolvimento científico e tecnológico criando a infra-estrutura necessária para estender o desenvolvimento industrial às regiões atualmente menos favorecidas. Face às limitações com que essa medida vem sendo cumprida, seria desejável que o governo federal, sem prejuízo da alocação de outros recursos para pesquisa e desenvolvimento nos estados, contribuísse na forma de parceria, para o orçamento das fundações e fundos estaduais como estratégia para acelerar a descentralização e criar, nas diversas regiões, condições para a implantação de programas em apoio da cooperação de universidades e centros de pesquisa com o setor produtivo. Na falta de infra-estrutura científica adequada e de recursos humanos atualizados e diversificados, parece inevitável que as medidas em favor da pesquisa e desenvolvimento em apoio à inovação e competitividade se concentrem, cada vez mais, nas regiões favorecidas e os desequilíbrios regionais tendem a se agravar.

\section{Notas}

$1 \mathrm{O}$ artigo 174 da Constituição Federal de 1946 dizia: "O amparo à cultura é dever do Estado - Parágrafo único -: O Estado promoverá a criação de institutos de pesquisa junto aos estabelecimentos de ensino superior".

2 Dos deputados Caio Prado Junior e Lincoln Feliciano

3 Instituídos por Jorge Americano, reitor da USP, em 1942, durante a Segunda Guerra, com o objetivo de financiar projetos ligados à defesa nacional.

4 Lineu Prestes

5 Adhemar de Barros (1947-1950).

6 Lucas Nogueira Garcez (1951-1954).

7 Jânio Quadros (1955-1958).

8 A Associação foi criada como reação à queda progressiva dos recursos que vinham sendo atribuídos à Universidade e das atitudes agressivas e hostis do governador para com vários docentes. 
9 A comissão era formada por cinco secretários de estado (Fazenda, Agricultura, Saú de, Educação e Viação), o reitor da USP, Antonio Barros de Ulhoa Cintra, Paulo Emílio Vanzolini, assessor do Secretário de Agricultura, Breno Asprino, assessor jurídico e Hélio Bicudo, sub-chefe da Casa Civil.

10 PETRUCCI, V. L. Condicionamento da trajetória institucional da Fundação de Ampaparo à Pesquisa do Estado de São Paulo. Tese de Mestrado, Unicamp, 1993.

11 Além de Ulhoa Cintra como presidente, o primeiro Conselho Supeiror era integrado por Alberto Carvalho da Silva, Carlos da Silva Lacaz, Crodowaldo Pavan, Enzo Azzi, Florestan Fernandes, Frederico Pimentel Gomes; José Ulpiano de Almeida Prado, Luiz Carlos Ulhoa Junqueira, Octavio Gaspar Ricardo, Paulo Emílio Vanzolini e Paulus Aulus Pompéia.

12 Jayme Arcoverde de Albuquerque Cavalcanti, diretor presidente; Warwick Estevãm Kerr, diretor científico; Raphael Ribeiro da Silva, diretor administrativo.

13 A proposta do Conselho Superior foi de que fossem transferidos os recursos desde 1947, ano em que se iniciou a vinculação de recursos na Constituição do Estado.

14 Na organização da Diretoria Científica Kerr valeu-se de observações feitas em visitas a várias instituições no Exterior, a convite da Fundação Rockefeller.

15 No total de auxílios estão incluídos iniciativas, projetos especiais, projetos temáticos, auxílios em apoio do desenvolvimento e inovação na empresa, e para recuperação e modernização da infra-estrutura, até 31 de dezembro de 1995. Não se incluem bolsas e auxílios aprovados em 1996.

16 (a) A comparação do desempenho posterior de 1.699 estudantes que, entre 1970 e 1982, tiveram bolsa de iniciação científica pela Fundação (Grupo A) e 1.072 estudantes que, no mesmo período, pleitearam a bolsa mas não foram atendidos (Grupo B), revelou, entre outros, os seguintes resultados:

- 30.8\% dos estudantes do Grupo A receberam posteriormente bolsa de mestrado da Fundação, em comparação com 13,3\% do Grupo B.

$-21,2 \%$ dos estudantes do Grupo A tiveram, posteriormente, auxílios aprovados pela Fundação contra $13,3 \%$ no grupo B.

- 31,5\% dos bolsistas de mestrado da Fundação, que vinham do Grupo A, tiveram bolsa de doutorado aprovada pela Fundação, contra 16,7\% dos que vinham do Grupo B.

(b) Em um grupo de 40 físicos formados no Instituto de Física da USP que receberam bolsas de mestrado e doutorado da FAPESP, 17 haviam pertencido ao Grupo A e 23 ao Grupo B, entre 1970 e 1975 . Os 40 nomes foram submetidos, sem informações quanto ao seu histórico, a um painel de 22 pesquisadores ativos em pesquisa em cinco estados, solicitando-se que os classificassem em uma das cinco categorias decrescentes quanto ao seu desempenho científico. Dos 17 com IC, 11 (64.5\%) foram classificados na categoria $\mathrm{A}$ (cientista produtivo com projeção internacional) contra quatro $(17,4 \%)$ entre os 23 com IC. 
17 Entre as diversas categorias de auxílios, para o período de 1977 a 1995, a que mostra maiores índices de aprovação é convite a visitantes do exterior com a média de 79.0\%; os piores índices são para Participação em Reuniões no Exterior (47.4\%) e no Brasil (56.9\%). Entre as bolsas no país, para o mesmo período, predominam doutorado e pós-doutorado, respectivamente com $83.5 \%$ e $73.9 \%$, e o pior desempenho é para Aperfeiçoamento, com $34.9 \%$. A partir de 1980, quando as bolsas no exterior foram separadas em doutorado e pós-doutorado, os respectivos índices de aprovação foram $33.7 \%$ (21.3\% a $45.7 \%)$ para doutorado e $68.1 \%$ (66.1\% e $78.9 \%)$ para pós-doutorado.

18 Outras iniciativas foram: centro de estudos sobre histologia comparada em mamíferos silvestres; estudos sobre Stevia rebaudiana; arquivo de fotografias aéreas; laboratório de produtos naturais; índice de localidades no mapa do IBGE; distribuição de sementes de vicia gramínea; centro de estudos de materiais para divulgação científica; apoio ao ensino de ciências; duplicação, construção e aperfeiçoamento de protótipos de equipamentos de pesquisa; doação de livros científicos; importação de compostos radioativos para pesquisa; contratação de especialistas estrangeiros.

19 Atualmente, Universidade Federal de São Paulo.

20 A Fundação de Amparo à Pesquisa do Rio Grande do Sul (FAPERGS) aprovou em seu Programa de Estimulo à Interação de Grupos e Centros de Pesquisa com o Setor Empresarial, até dezembro de 1994, 94 propostas no total de R \$3,8 milhões, repartidos entre empresas (31\%) e Fundação (69,0\%). A Fundação de Amparo à Ciência e Tecnologia de Pernambuco (FACEPE), adotou, em 1995, um Programa de Capacitação Tecnológica da Empresa de Base Local. Em 1994 a Fundação de Amparo à Pesquisas de Minas Gerais (FAPEMIG) destinou R\$ 2.5 milhões a quatro entidades estaduais de pesquisa aplicada - CETEC, FUNED, EPAMID e FJP.

21 Até 1990, o cálculo exato do valor em dólares das quantias transferidas é muito impreciso, em virtude dos altos índices de inflação e, até 1984, de variações na freqüência anual e datas de tranferências.

22 Entre 1962 e 1971, essas mesmas áreas foram as que receberam mais recursos e so$\operatorname{mam} 79,4 \%$.

23 As outras cinco áreas, no período, foram: Geociências, 4,57\%; Matemática e Estatística, 3.33\%; Economia e Administração, 1.10\%; Astronomia e Ciências Espaciais, 1.07\%; Arquitetura e Urbanismo, $0.64 \%$. Os 7,1 restantes, correm por conta de atividades comuns a todas as áreas (projetos interdisciplinares, publicações, simpósios, especialistas estrangeiros, rede ANSP).

24 O parágrafo de autoria do deputado federal Florestan Fernandes, membro do primeiro Conselho Superior da FAPESP, de 1961 a 1963, estabelece que é facultado aos estados e ao Distrito Federal vincular parcela de sua receita orçamentária a entidades públicas de fomento à pesquisa científica e tecnológica.

25 Receita dos impostos (CE-AL); receita líquida dos impostos (RS); receita tributária (AM, BA, MS, GO, MT, PR, RJ, SP, SE, TO); receita corrente (MA, MG, SC); receita orçamentária (DF, ES, PA, PI, PB, PE); receita estimada (AC). A distribuição de percentuais é: MG, GO, AM, 3\%; ES, 2.5\%; RJ, AL, PA, CE, MT, 2\%; RS, MS, BA, 1.5\%; PE, SP, PB,SC, PI, 1\%; MT, TO, SE, 0.5\%; PA, DF, 0.3\%; AC, percentual não definido. 
26 Ver, por exemplo: As Fundações estaduais e financiamento da pesquisa e pós-graduação no Brasil - Estudo apresentado ao Ministério de Ciência e Tecnologia em 1990, por uma comissão de pesquisadores (mimeo); Fomento à Pesquisa e Entidades Estaduais, Gerson Ferreira Filho, Abílio Baeta Neves e Luiz Fernando Candiota, com a colaboração da SBPC (mimeo). Participação das Fundações Estaduais de Amparo à Pesquisa - FAPs na Gestão dos Recursos do PADCT e na Operação de seus Programas, Relatório de Consultoria, Alberto Carvalho da Silva, 1995.

Alberto Carvalho da Silva, médico, é professor honorário do Instituto de Estudos Avançados da USP.

Estudos AvanÇADOS 10 (28), 1996 